\title{
Improvement of a pharmaceutical enterprise's business processes at the stage of preclinical development of new drugs
}

\author{
Mikhail V. Belova
}

E-mail: mbelov@ibs.ru

Mikhail A. Shakhmuradyan ${ }^{\mathrm{b}}$

E-mail:mshakhmuradyan@econ.msu.ru

${ }^{a}$ IBS Group Holding Ltd.

Address: 3 build. 1, Skladochnaya Street, Moscow 127018, Russia

${ }^{\mathrm{b}}$ Lomonosov Moscow State University

Address: 1 build. 46, GSP-1, Leninskie Gory, Moscow 119991, Russia

\begin{abstract}
The rapid increase of the population, as well as the high rate of urbanization, are leading to an increased need for medical supplies, as well as the need for faster release of new drugs to the market. In this article, the authors analyze and aggregate the activities of a number of pharmaceutical companies at the preclinical stage of drug development to identify optimal management models at the stage of each operational iteration. The work methodology is based on an empirical study based on two progressive stages: a qualitative analysis of the business processes of pharmaceutical enterprises, reflected in regulatory rules and company reports, as well as in-depth interviews with representatives of these commercial organizations. Based on the results obtained at the end of the first stage, the authors established the problem of the lack of a unified presentation of standardized operating procedures, as well as an aggregated representation of the stage of research work on the production of the drug. When considering each business process of this stage, the authors presented a model of the minimum unit of integrated activity (MUnIA). This model most optimally describes local operational business processes during drug development, and can also serve as an instrumental framework for guiding preclinical studies in the formation of a document on standardization of operational procedures. The results of the first stage of the empirical analysis were verified during the second part of the work in-depth interviews with industry representatives. The findings of this study can be used by project managers at the preclinical testing stage to reduce the time spent on operating procedures.
\end{abstract}




\section{Graphical abstract}

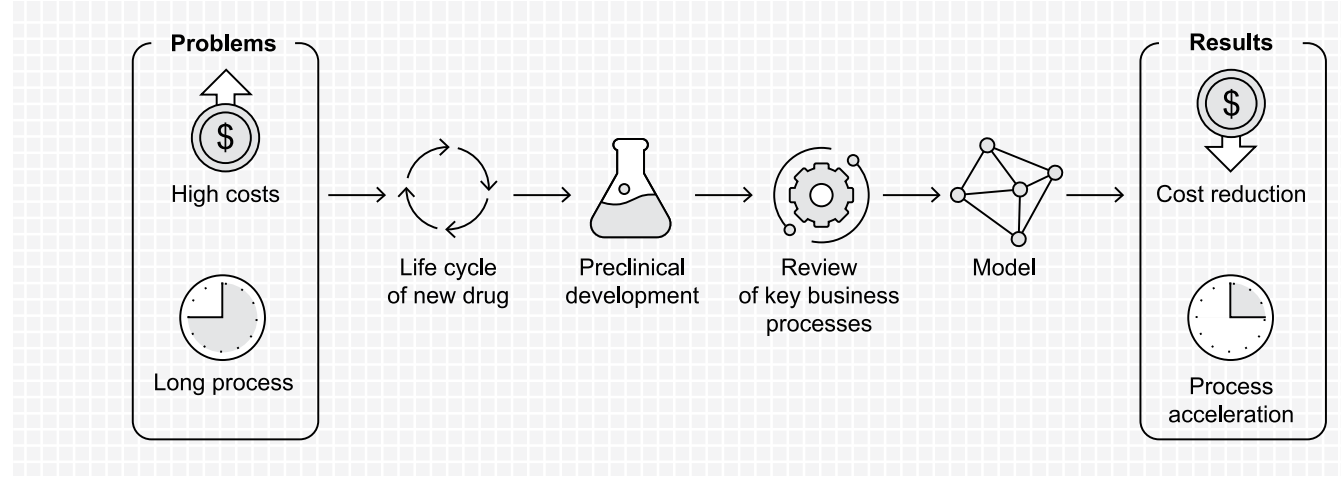

Key words: business process; pharmaceuticals; pharmaceutical company; digitalization; project management.

Citation: Belov M.V., Shakhmuradyan M.A. (2019) Improvement of a pharmaceutical enterprise's business processes at the stage of preclinical development of new drugs. Business Informatics, vol. 13, no 4, pp. 17-27. DOI: 10.17323/1998-0663.2019.4.17.27

\section{Introduction}

7 oday the international pharmaceutical industry is undergoing significant changes. Due to the increase in the number of able-bodied people, the increase in general financial well-being, and the strengthening of the health policy of developed countries, the demand for pharmacological preparations is steadily increasing, while the costs of medicines are only going up [1]. The potential decline in the financial results of a modern pharmaceutical enterprise is explained not only by a high investment threshold for entering this market, but also by general competition, which requires the constant implementation of comprehensive innovative management strategies to maximize the product's life value by improving the current operating activities of the enterprise. Today in the world market, it is customary to single out several types of pharmaceutical organizations. The main types of pharmaceutical com- panies, classified by main type of activity [2], are presented in Table 1.

We will consider the business processes of the first type of pharmaceutical enterprise, including a full cycle of creation, testing and implementation of the product. The aim of the study is to analyze the business processes of the pharmaceutical company at the preclinical research stage, with the subsequent proposal of an informed solution in terms of their improvement.

The article has the following structure. First of all, theoretical provisions are considered by describing the full life cycle of drug development and production. What follows are the results of the analysis of business processes at the stage of preclinical trials of the drug, with the identification of a potential area of operational process management. Finally, we formulate the results of summarizing the systemic processes of pharmaceutical company research work and we present the model verified through interviews with managers of pharmaceutical companies. 


\section{The main types of pharmaceutical companies classified by main type of activity}

\begin{tabular}{c|l|l} 
No & \multicolumn{1}{|c|}{ Type of pharmaceutical company } & \multicolumn{1}{c}{ Examples } \\
1. & $\begin{array}{l}\text { Full circle companies whose activities include partici } \\
\text { pation in the entire product life cycle from the development } \\
\text { and research of the molecule to the marketing }\end{array}$ & $\begin{array}{l}\text { HTC «ChemRar», Roche Holding, Novartis } \\
\text { International AG, Pfizer Inc., GlaxoSmithKline plc, } \\
\text { Sanofi, etc. }\end{array}$ \\
\hline 2. & R\&D enterprises & $\begin{array}{l}\text { Research institute «ChemRar», Research institute } \\
\text { of Chemical Diversity, Insilico Medicine Inc., etc. }\end{array}$ \\
\hline 3. & Manufacturing companies & $\begin{array}{l}\text { «ChemRar» Research institute of Chemical } \\
\text { Diversity, Biolntegrator, Sanofi-Aventis East, etc. }\end{array}$ \\
\hline 4. & Distribution companies & $\begin{array}{l}\text { Roche Moscow, Bioritm, Dileo-pharma, PHARMA } \\
\text { Distribution Group, etc. }\end{array}$ \\
\hline
\end{tabular}

\section{The life cycle of drug development and production}

To consider the main business processes of a modern full-cycle pharmaceutical enterprise, it is necessary to determine the subject of activity for the production of a medicinal product. In this study, the subject of activity is drug development - the process by which potential medicines are discovered or developed. The process of discovery drugs today has an established theoretical and methodological base and includes two main phases - preclini- cal research (on average, 3 years) and clinical trials (from 6 years or more) [3-4].

Since the demand for new methods of pharmacological treatment remains high, and the time required to bring new drugs to the market is about 10 years, pharmaceutical enterprises are looking for different ways to shorten the life cycle of drugs at different stages. On average, to develop and obtain approval for a new drug, a full-cycle commercial enterprise needs about $\$ 2.6$ billion [5]. If we consider the financial aspects (Figure 1), it can be noted that business processes for the production of drugs at

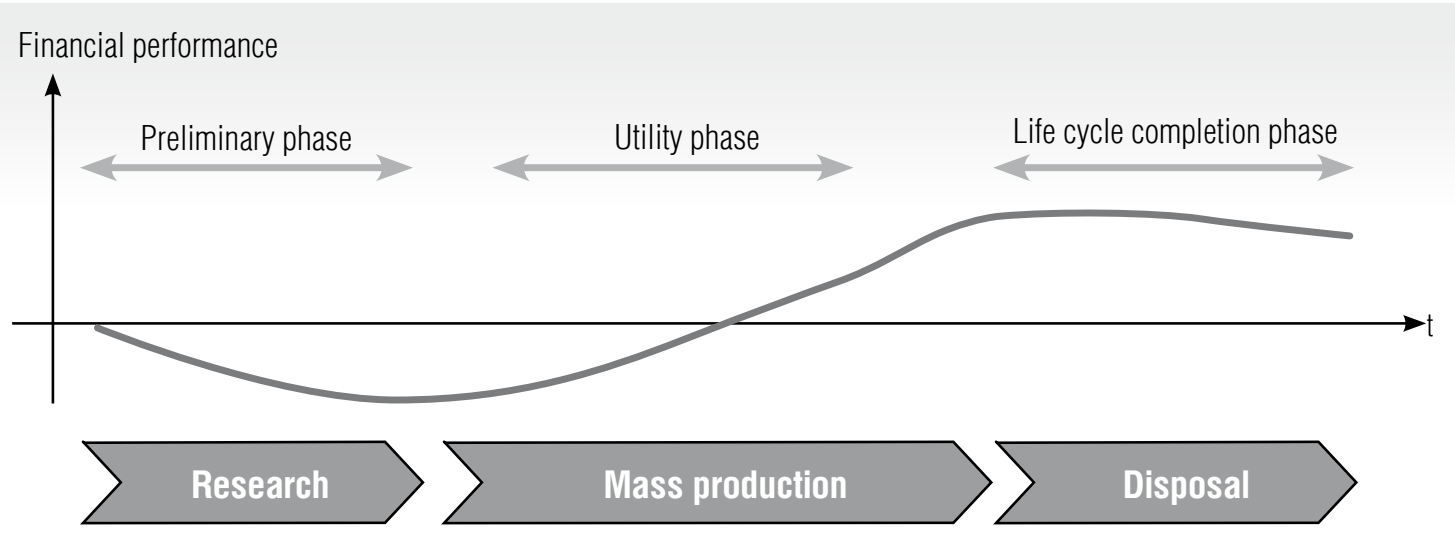

Fig. 1. Financial performance of the drug life cycle stages 
the stage of research in the preliminary phase, together with the average cost of preclinical studies, characterize the period most unfavorable for the investor. In order to improve operational activities and shorten the life cycle of drug development, the authors conducted a comprehensive analysis of the business processes of pharmaceutical enterprises reflected in regulatory documents and reports of a number of international pharmaceutical companies.

In this work, the business process is defined as a set of tasks that includes systems and methods applied to create and develop the final product or service provided to the buyer [6]. Accordingly, for the possible improvement of business processes, it is necessary to decompose the entire production life cycle of the drug and determine processes that could be systematized to reduce time.

The life cycle of the production and development of drugs in the early stages involves a marketing analysis, which is carried out by the relevant division of the company. During such analysis, current and future demand is taken into account, and a proposal is formulated to create a specific drug or treat a specific disease. Before making a decision, the company's management can analyze the current produc- tion and research capacities to assess the possibility of working on the projects proposed in the plan.

At the time of approval, the ultimate goal of drug development, research leaders who organize and control the preclinical stages (target search, agent search, prototype optimization, drug candidate testing) and clinical trials (Figure 2) are appointed. The clinical trials of a drug take the largest part of the drug development time. At the same time, due to regulatory reasons, at the level of the company it is not possible to accelerate this stage.

The process of movement from preclinical trials to clinical trials can be described by the next steps. A pharmaceutical company is applying for a clinical trial authorization (CTA), which is being reviewed by a number of experts. After that, a decision is made about the possibility of conducting tests on humans.

At phase 1 of the trial, the safety and pharmacology of the candidate drug are tested on a small group of healthy volunteers (from 20 to 100 people) who are given small doses of the compound. Sometimes phase 0 or "proof-ofconcept" $(\mathrm{PoC})$ is performed, when a candidate for drugs is tested in a small group of patients (from 5 to 15 ) to determine the "mechanism of action" of the drug in the human body.

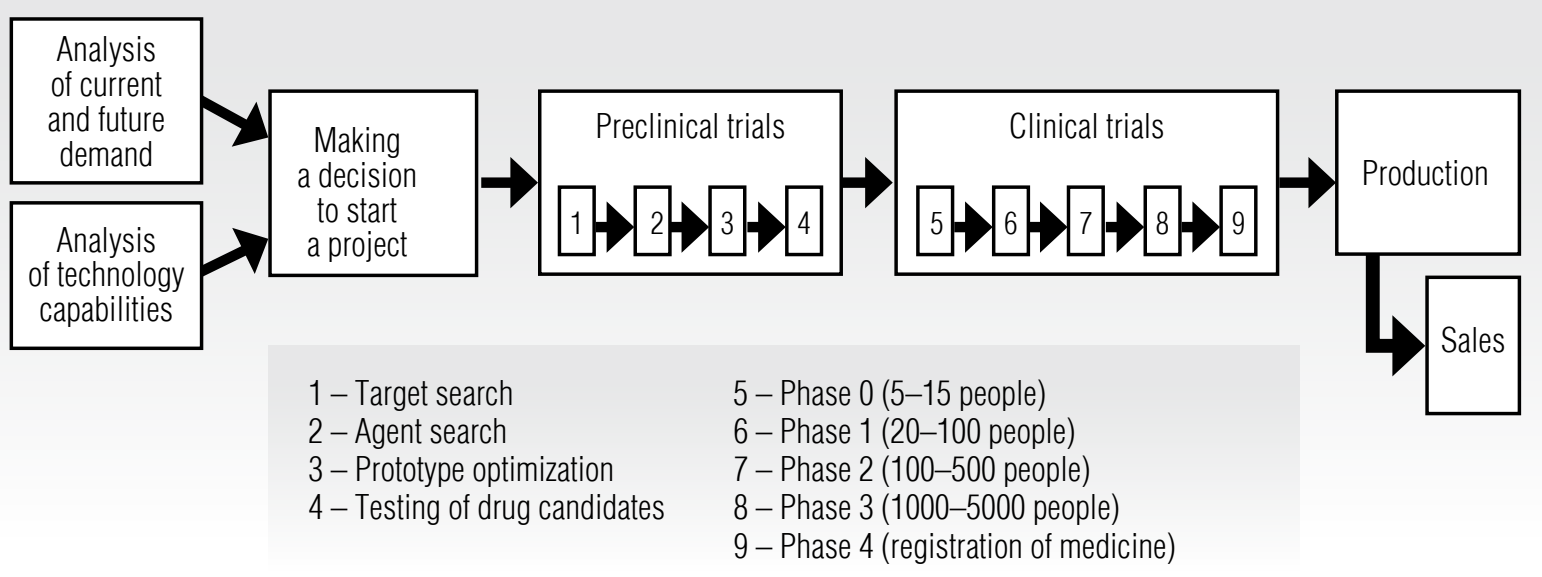

Fig. 2. Main stages of the drug life cycle of a full cycle pharmaceutical company 
At phase 2, the effectiveness of the compound is studied on a group of volunteers who have a condition for the treatment of which the drug is directed. Typically, such a group includes from 100 to 500 patients whose vital indicators are constantly monitored and evaluated. The purpose of phase 2 is to determine the most effective dose and method of drug delivery (for example, orally or intravenously). Most drugs that fail during clinical trials due to their inefficiency and insecurity are detected at this phase.

At phase 3, potential drugs are tested in a significantly larger population (from 1000 to 5000 people), in several international centers. At the same time, the pharmaceutical company needs to collect sufficient data on the safety and efficacy of drugs in order to apply for licensing to the regulatory authority (for example, in the UK it is The Medicines and Healthcare Products Regulatory Agency and in the USA is The Food and Drug Administration).

At phase 4, the drug is finally approved by the national supervisor and production is launched with the simultaneous determination of distribution channels.

The listed stages are normatively defined and there are mandatory procedures which to identify the safety of the drug. In modern conditions, the pharmaceutical industry is forced to transform and improve precisely the stage of preclinical development in order to compensate for the longer wait for human trials and approval of regulatory authorities. Therefore, given the regulatory requirements of the relevant regulatory authorities, it seems important to consider the process of drug development precisely at the stage of research work.

\section{The business processes at the preclinical stage of drug development}

A significant part of the work on the identification and development of drugs in the early stages is carried out by universities and research institutes, the activities of which are supervised and controlled by project managers of full circle companies [7]. For example, in a university laboratory, scientists with the support of grants from research institutions or of a pharmaceutical company conduct basic research to determine the cause of the disease. The interaction of several entities in the development of drugs is an integral part of the business processes of a modern international pharmaceutical company. Under these conditions, not only the technological or qualification level of the performers, but also the coordination and regulation of their interaction have a significant impact on the progress of research.

The activity of project managers at the preclinical stage is determined not only by corporate standards, but also by external standards [8]. In particular, an internal preclinical testing processes are governed by such standards as the "good laboratory practice" (GLP) system created in the USA, Russian GOST 33647-2015, as well as GAMP (good automated manufacturing practice) and ISO / IEC 17025: 2005 (in the Russian Federation - GOST ISO / IEC 17025-2009) [9]. Based on the "principles of good laboratory practice," the planning and implementation of key stages of research work is carried out, and the reporting form for each iteration is also regulated. The main purpose of these activities is to study the safety of a chemical. Each hierarchical level is regulated by a number of norms; however, there is an area of operational procedures, the development of which is under the supervision of research leaders. So, in accordance with GOST 336472015, each unit of the testing center should have its own standard operating procedures (SOP) for the type of activity regulated by the administration; however, there is no unified presentation of the document in the form of a normative act [10]. In addition, GOST ISO / IEC 17025-2009 obliges the administrative unit organizing or hiring outside research to 
develop and provide a management system in accordance with its field of activity. This management system should be documented in the form of a detailed description of the system, program, procedures and instructions to the extent necessary for a qualitative reflection of the research results [9]. In addition, the project manager should monitor not only the organization of security of operational processes, but also the documentation of the security of the compound - the drug candidate. In accordance with ICH Guideline M3 (R2), approved by the International Council for the Harmonization, preclinical safety studies should be sufficient to characterize potential side effects that may occur in a supported clinical trial.

Based on the foregoing, the authors concluded that there is a large load on project managers at the preclinical stage of drug development. Nevertheless, one of the most important aspects determining the increase in time for operating procedures may be the lack of a standardized SOP [11-12]. Based on this, the following is considered the problem of finding an improved way to organize operational activities in the framework of preclinical trials.

Based on a qualitative study of regulatory documents (in particular, GOST 33647-2015 and ISO / IEC 17025: 2005), as well as business processes of a number of pharmaceutical companies, the authors described a number of operational procedures that take place at the stage of drug discovery and development (Figure 3). It should be noted that modern research is entirely conducted at the molecular level. The stage of target search is a priority for preclinical studies. The target can be a gene, protein, or protein-protein interactions (PPIs), either contributing to the disease, or can interfere with treatment, for example, by blocking the desired receptor.

Since some diseases are associated with dysfunction of not only one molecule, but, for example, 5-10 bound proteins, the scientific team needs to study at least five times more targets. Once a potential target has been identified, the researchers will move on to finding a compound that will act on this goal and, therefore, can affect the disease. Some 10000 or more compounds can be considered. These are usually reduced to $10-20$, which, according to the forecasts of the working group, can affect the disease. Currently, the process of finding a new drug against a selected target for a specific disease usually includes molecular docking (molecular modeling to find the optimal archi-

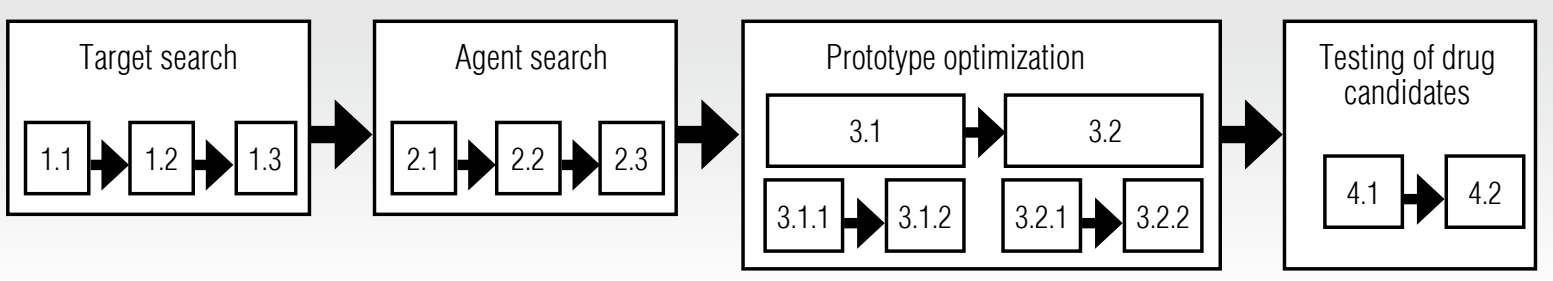

1.1. Determination of the number of targets

1.2. Target type determination

1.3. Target validation

2.1. Virtual screening

2.2. High performance screening

2.3. Estimation of ADME
3.1. Analysis by a group of molecular biologists

3.1.1. Modeling

3.1.2. Pharmacology / Pharmacokinetics / Toxicology

3.2. Analysis by a group of chemists

3.2.1. Modeling

3.2.2. Pharmacology / Pharmacokinetics / Toxicology
4.1. In vitro tests

4.2. In vivo tests

Fig. 3. Aggregated scheme of the research phase 
tecture in the target protein binding center), virtual screening (automated selection of the necessary chemical compounds from specialized databases), as well as high-throughput screening (high-throughput screening), in which large combinatorial libraries of chemicals are tested for their ability to inhibit the target.

After determining the relevant compounds, their pharmacokinetic verification (ADME) is performed. The next step is the optimization of prototypes, which is carried out in parallel by a group of molecular biologists, as well as a team of specialists in the field of medical chemistry. The activities of these two groups are also supervised by the project manager. After successful selection and verification of a number of compounds, in vitro and in vivo experiments are carried out. In order to gain access to the clinical phase, the project team needs to carry out the following studies: pharmacological (primary pharmacodynamics, secondary pharmacodynamics, pharmacological safety, pharmacodynamic drug interactions) and pharmacokinetic (absorption, distribution, metabolism, excretion and toxicological properties of the drug, pharmacokinetic drug interaction).

During the study of general toxic properties (assessment of acute and chronic toxicity), on the one hand, toxic doses that can cause the death of animals are revealed, and thereby determine the dose limit that cannot be exceeded. On the other hand, the safety of therapeutic doses with prolonged exposure to animals is assessed. For this, the drug is administered daily both in therapeutic doses and in doses ten times higher than therapeutic. The duration of the experiment depends on the duration of the intended course in clinical practice. During the experiment, the functional state of all body systems, the weight and behavior of animals are analyzed. After completing the course, a histological examination of all organs and tissues is performed. Also, in the framework of a safety assessment at the preclinical stage, experiments are conducted on the so-called specific toxic- ity. In general, these studies make it possible to assess the possible effect of the studied drug on the immune status of the body, the reproductive system and offspring, the genetic apparatus, as well as the possibility of allergic reactions and provoking malignant tumors.

However, these studies cannot provide reliable information on the effect of the studied drugs on humans, since the organism of laboratory animals differs from the human one both in pharmacokinetic characteristics and in the response of organs and systems to drugs. That is why it is necessary to conduct clinical trials of drugs. Thus, the main result of preclinical studies of a new drug is a prediction of its safety for humans.

\section{The model of the minimum unit of integrated activity (MUnIA)}

The aspects we have considered explain the importance of a detailed analysis of processes at all stages of research work. As already noted, industry actors are looking for more effective approaches to launching new products on the market that can accelerate product development while reducing operating costs. A detailed analysis of the processes of preclinical studies allows us to draw conclusions about the possibility of a unified and improved presentation of business processes by introducing the concept of a minimum unit of integrated activity (MUnIA). Such a presentation of the MUnIA in the context of the analysis of pharmaceutical enterprises business processes reflects the cyclical nature of the relevant work (Figure 4).

Any operational process at the preclinical stage begins with the formation of many hypothetical constructs that need to be verified. Verification of the generated hypotheses is a mandatory and logical continuation of the systematic process of operational activity. If it is impossible to move from one stage to another, validation is carried out at the stage of hypothesis testing (small cycle), and in case of repeated failure of the transition, a return to the stage of formation of many hypotheses for repeated test- 


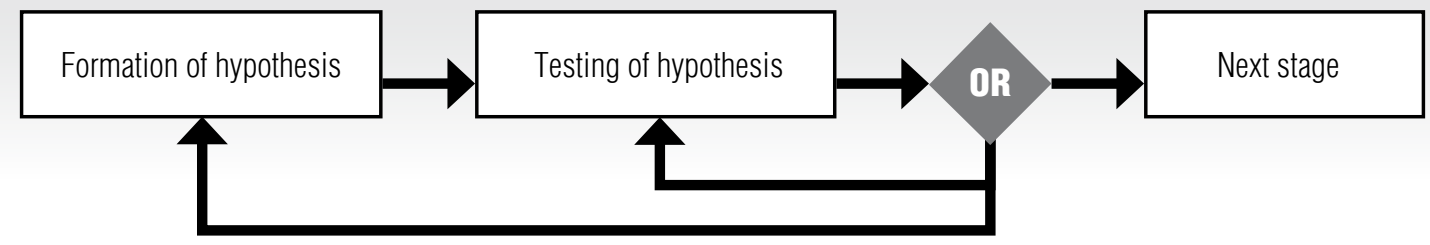

Fig. 4. The scheme of the minimum unit of integrated activity (MUnIA)

ing (large cycle). This cyclical design not only reflects the minimum systematic process of integrated research activities for the creation and development of drugs, but also associates entities with added value, which can worsen or otherwise prevent the fulfillment of the intended function of the business process. The main algorithm for working with a minimum unit of integrated activity is the complete decomposition of the business processes of a particular stage of research to an appropriate scale, the determination of the place of current activity in the model, the rationalization of the stage and subsequent improvement (adaptation to MUnIA cycles).

To demonstrate MUnIA applicability, the framework was simulated using the complex of preclinical stages of drug development as an example. So, at figure 5 each iteration of the stage of research work is schematically presented in accordance with the detailed decomposition and approbation of the model. The main methodology for verifying the applicability of the MUnIA was in-depth interviews with representatives of the pharmaceutical industry. At the same time, an in-depth interview is understood as an informal personal conversation with respondents, within the framework of previously agreed topics and structure.

In forming the sample of companies, the following criteria were used. First, the enterprise should be engaged in research and development of medicines. Secondly, the enterprise should be international and at the same time operate on the territory of Russia. Thirdly, the number of employees must be at least 1000 people. Thus, four companies were included in the study sample. The research design involved the participation of one to three managers from each enterprise.

The in-depth interviews on model verification and its application were conducted with five managers of Russian pharmaceutical companies. Respondents were selected taking into account their high awareness of the research activities of the organization. Interviews were conducted from August 1, 2019 to October 14, 2019. The duration of each interview ranged from 20 minutes to one hour; the average interview time was 35 minutes. Each respondent was asked 12 questions with specific answer options ("yes", "rather yes than no," "rather no than yes," "no") and two open questions. The first five questions clarified the activities and operational processes of the pharmaceutical company. The following three questions served to verify the main stages of the drug development life cycle. The last four questions clarified the concept of the proposed model and the possibility of its use in the operational process, namely: "Does the MUnIA model describe the minimum life system in the framework of preclinical studies?", "Is it possible to optimize operational procedures for preclinical tests using the MUnIA model?", "Is it possible to implement the MUnIA model in the preparation of SOPs?", "Is it possible to implement the MUnIA model in the content of the SOP of your company?" Two open questions were asked to determine directions for finalizing the approach to the analysis of business processes in general and the model: "what else should be taken into account in this approach to the analysis of business processes?" and "how can the MUnIA 
model be instrumentally integrated into the current information ecosystem of pharmaceutical enterprises?"

The MUnIA model was verified by all respondents (with certain conditions and improvements) as possible and recommended for use. A diagram was also formed (Figure 5), which stipulated the technical applicability and possibility of MUnIA implementation in operational business processes of the drug life cycle stage. For example, when considering such a sub stage of preclinical drug development as target search, it is necessary to consider that the initial goal is to determine the targets that will be affected by the compound, their number and type. In practice, the project's working group considers a specific disease by analyzing a cascade of proteins. This procedure is a stage in the formation of many hypotheses about specific interactions that determine deviations. This is followed by a direct verification of scientific assumptions about the potential types and number of candidates in the target, using appropriate software tools. With successful validation, a transition from processes $1.1 / 1.2$ (determination of the number of targets / determination of types

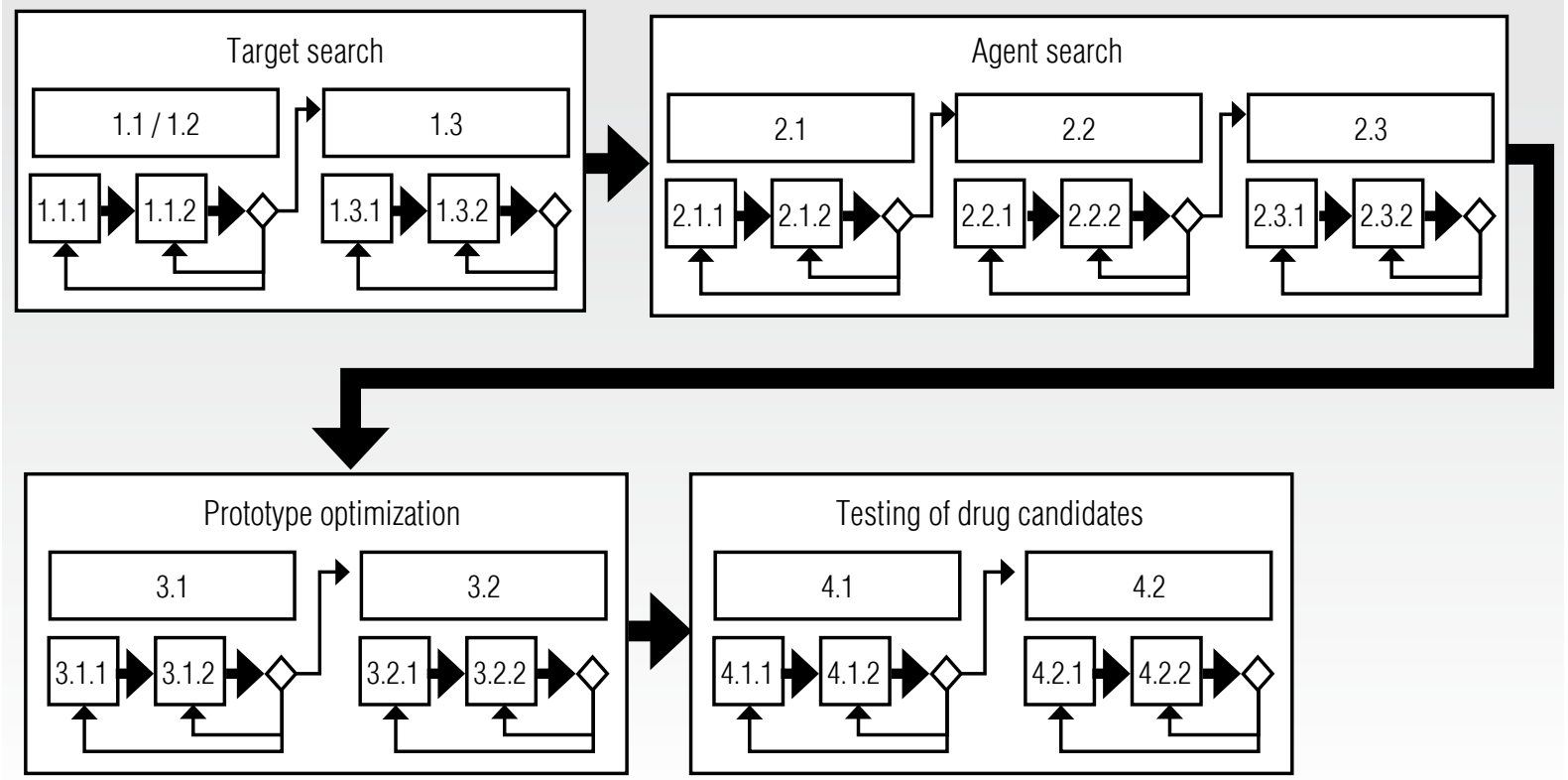

1.1. Determination of the number of targets

1.1.1. Protein cascade analysis

1.1.2. Testing protein interaction methods

1.2. Target type determination

1.3. Target validation

1.3.1. The formation of hypotheses about the relationship of the disease and targets

1.3.2. Proof of concept (PoC)

2.1. Virtual screening

2.1.1. Defining databases for library search

2.1.2. Selection of chemical libraries
2.2. High performance screening

2.2.1. Defining of test systems

2.2.2. Series testing

2.3. Estimation of ADME

2.3.1 Testing of hypothesis

2.3.2 Compounds testing

3.1. Analysis by a group of molecular biologists

3.1.1. Modeling

3.1.2. Pharmacology / Pharmacokinetics / Toxicology
3.2. Analysis by a group of chemists

3.2.1. Modeling

3.2.2. Pharmacology / Pharmacokinetics / Toxicology

4.1. In vitro tests

4.1.1. Formation of hypothesis

4.1.2. Testing of hypothesis

4.2. In vivo tests

4.2.1. Formation of hypothesis

4.2.2. Testing of hypothesis

Fig. 5. Preclinical operating procedures in accordance with the MUnIA 
of targets) to process 1.3 (validation of targets) occurs. If the outcome is negative, the study is revised as part of operation 1.1.2 (verification of protein interaction methods), as part of the small cycle of the MUnIA model. In the case of a repeated negative outcome, the analysis is redirected along a large cycle to process 1.1.1, where a new set of hypotheses is formed for further verification.

Similar testing of the model is possible at any of the sub stages of preclinical trials. Thus, MUnIA is a management framework that can not only affect the main operational and intermediate procedures, but also provide systematization when monitoring the current activity of drug product development by project managers. In general, respondents confirmed the possibility of using the MUnIA model to manage and improve the preclinical stage of the study, as well as the possibility of using the above scheme as a recommendation for a unified presentation of SOPs.

\section{Conclusion}

This article analyzes the complex business processes of some full-cycle pharmaceutical enterprises at the preclinical stage for their fur- ther improvement. Qualitative analysis is based on data presented in industry regulations, open information from a number of commercial pharmaceutical companies, and in-depth interviews with industry representatives. The authors aggregated and schematically presented the main business processes of a pharmaceutical enterprise of this type. Based on the compilation, description and subsequent decomposition of the drug's life cycle, the problem of the lack of a standard unified form of processes representation at each operational iteration of preclinical trials has been identified which is a potential source of increased production time. After that, the model of the minimum unit of integrated activity (MUnIA) was proposed; this can be considered as a fundamental unit of standard operating procedures that describe the sequence of actions for developing drugs. The model can be used by both drug discovery research managers and project managers.

Thus, the priorities for further research may be the quantitative confirmation of the MUnIA efficiency at the preclinical stage, as well as the analysis of options for potential scaling of the model to other stages of the life cycle of drug production.

\section{References}

1. Lichtenberg F.R. (2008) Have newer cardiovascular drugs reduced hospitalization? Evidence from longitudinal country-level data on 20 OECD Countries, 1995-2003. The National Bureau of Economic Research. Available at: https://www.nber.org/papers/w14008 (accessed 30 June 2019).

2. Pilnikova E.G. (2016) Features of the activities of pharmaceutical manufacturing companies in modern conditions. Business Education in the Knowledge Economy, no 1, pp. 61-64 (in Russian).

3. Golovko A.S., Golovko Yu.S., Ivashkevich O.A. (2012) Modern methods of searching for new drugs. Bulletin of BSU. Series 2: Chemistry, Biology, Geography, no 1, pp. 7-15 (in Russian).

4. PhRMA (2015) Biopharmaceutical research \& development: The process behind new medicines. Available at: http://phrma-docs.phrma.org/sites/default/files/pdf/rd_brochure_022307.pdf (accessed 23 June 2019).

5. Tufts University (2016) Outlook 2016. Tufts Center for the Study of Drug Development. Available at: https://static1.squarespace.com/static/5a9eb0c8e2ccd1158288d8dc/t/5aa2fc 9d0852297555747051/1520630944033/Outlook-2016.pdf (accessed 23 June 2019).

6. Locuson C. (2016) Project management in drug discovery: Current practices and opportunities. Project Management. Available at: https://www.projectmanagement.com/articles/331399/Project-Managementin-Drug-Discovery--Current-Practices-and-Opportunities (accessed 23.06.2019). 
7. Frearson J., Wyatt P. (2019) Drug discovery in academia - the third way? Expert Opinion on Drug Discovery, vol. 5, no 10, pp. 909-919. DOI: 10.1517/17460441.2010.506508.

8. Raman A., Tok W.H. (2018) A developer's guide to building AI applications. Create your first intelligent bot with Microsoft AI. Sebastopol, CA: O’Reilly Media.

9. GOST ISO/IEC 17025-2009. General requirements for the competence of testing and calibration laboratories. Available at: http://docs.cntd.ru/document/gost-iso-mek-17025-2009 (accessed 31 July 2019) (in Russian).

10. GOST 33647-2015. Principles of good laboratory practice (GLP). Terms and definitions. Available at: http://docs.cntd.ru/document/1200129061 (accessed 31 July 2019) (in Russian).

11. Chen J., Luo X., Qiu H., Mackey V., Sun L., Ouyang X. (2018) Drug discovery and drug marketing with the critical roles of modern administration. American Journal of Translational Research, vol. 10, no 12, pp. 4302-4312.

12. Pattanaik A. (2014) Complexity of project management in the pharmaceutical industry. Proceedings of the PMI Global Congress 2014, Dubai, United Arab Emirates, 5-7 May 2014. Available at: https://www.pmi.org/learning/library/project-management-complexity-pharmaceutical-industry-1487 (accessed 23 June 2019).

\section{About the authors}

\section{Mikhail V. Belov}

Cand. Sci. (Tech.);

Deputy CEO, IBS Group Holding Ltd., 3 build. 1, Skladochnaya Street, Moscow 127018, Russia;

Head of the Department of Information Business Systems, NUST MISIS,

8/11, Maly Tolmachevsky Lane, Moscow 119017, Russia;

E-mail: mbelov@ibs.ru

\section{Mikhail A. Shakhmuradyan}

Doctoral Student, Department of Economics of Innovation, Faculty of Economics, Lomonosov Moscow State University,

1 build. 46, GSP-1, Leninskie Gory, Moscow 119991, Russia;

E-mail: mshakhmuradyan@econ.msu.ru 